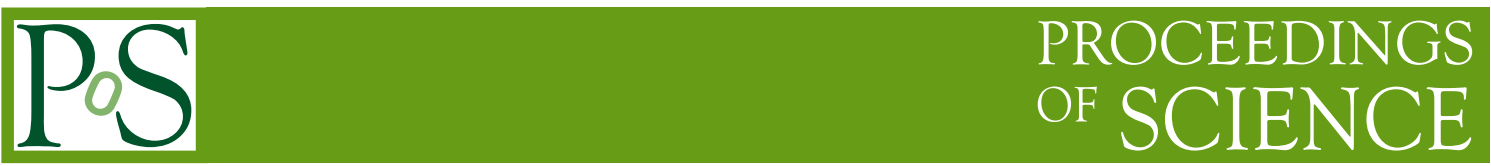

\title{
The upgrade of the ALICE Inner Tracking System
}

\author{
Ivan Ravasenga*t on behalf of the ALICE collaboration \\ Politecnico di Torino and I.N.F.N. \\ E-mail: ivan.ravasenga@polito.it
}

In 2021, for the third run of the CERN Large Hadron Collider (LHC), Pb-Pb collisions will be performed at a centre-of-mass energy per nucleon of $5.5 \mathrm{TeV}$, with an integrated luminosity of $6 \times 10^{27} \mathrm{~cm}^{-2} \mathrm{~s}^{-1}$ and at an unprecedented interaction rate up to $50 \mathrm{kHz}$.

To fulfil the requirements of the ALICE physics program for Run 3, the ALICE experiment at LHC is planning a major upgrade during the Long Shutdown 2 of LHC in 2019-2020. One of the key elements, is the construction of a new ultra-light and high-resolution Inner Tracking System (ITS). The upgraded ITS will significantly enhance the determination of the distance of closest approach to the primary vertex, the tracking efficiency at low transverse momenta, and the read-out rate capabilities, with respect to what can be achieved with the current detector. It will consist of seven layers equipped with silicon Monolithic Active Pixel Sensors (MAPS) with a pixel size of the order of $30 \times 30 \mu \mathrm{m}^{2}$. They will be produced by Towerjazz with its $0.18 \mu \mathrm{m}$ CMOS Imaging process. To transmit (receive) data to (from) the sensors, Flex Printed Circuits (FPCs) will be wire-bonded to the sensors themselves.

One of the main physics goals consist in improving the reconstruction capabilities of heavyflavour mesons and baryons. In addition, the new tracking detector will allow us to study lowmass dileptons and low- $p_{T}$ charmonia at mid-rapidities.

55th International Winter Meeting on Nuclear Physics

23-27 January, 2017

Bormio, Italy

\footnotetext{
${ }^{*}$ Speaker.

${ }^{\dagger}$ Dipartimento DISAT del Politecnico and Sezione INFN, Turin, Italy
} 


\section{Introduction}

The ALICE Collaboration at the CERN Large Hadron Collider (LHC) is building a major upgrade of the experimental apparatus during the Long Shutdown 2 (LS2) of LHC in 2019-2020. The upgrade aims at enhancing the physics capabilities of ALICE for the measurements of rare probes on a wide range of transverse momenta in $\mathrm{pp}, \mathrm{p}-\mathrm{Pb}$ and $\mathrm{Pb}-\mathrm{Pb}$ collisions. A target integrated luminosity of $10 \mathrm{nb}^{-1}$ in $\mathrm{Pb}-\mathrm{Pb}$ collisions at a centre-of-mass energy per nucleon of $5.5 \mathrm{TeV}$ is foreseen.

The physics goal consists in reaching high-precision measurements of the Quark-Gluon Plasma (QGP) state, studying heavy-flavour quarkonia at very low $p_{T}$, vector mesons and low-mass dileptons, as well as light nuclei and hyper-nuclei.

In order to reach its physics objectives, ALICE planned the upgrade of many detectors included the Inner Tracking System (ITS), the Time Projection Chamber (TPC) by introducing Gas-Electron Multipliers (GEM), a new Muon Forward Tracker (MFT) to add vertexing capabilities to the current Muon Spectrometer and, the upgrade of the Online and Offline Systems $\left(\mathrm{O}^{2}\right.$ project) [1].

This article presents an overall description of the upgrade of the ALICE ITS (Sec. 2, 3 and 4) that plays a key-role for the enhancement of the physics capabilities relaying on the determination of the distance of closest approach to the primary vertex, the tracking efficiency at low transverse momenta $(<1 \mathrm{GeV} / c)$, and the read-out rate, with respect to what can be achieved with the current detector. The expected performance of the upgraded ITS will be illustrated in Sec. 5 with different examples.

A complete description of the ITS upgrade project is given in the ITS Technical Design Report [2].

\section{ALICE Inner Tracking System}

The present ALICE ITS is composed by six layers of silicon detectors: two inner layers of Silicon Pixel Detectors (SPD), two middle layers of Silicon Drift Detectors (SDD) and two outer layers of Silicon Strip Detectors (SSD).

The present technology fully meets the design requirements, however in order to cope with the future increase of the LHC luminosity (up to $6 \times 10^{27} \mathrm{~cm}^{-2} \mathrm{~s}^{-1}$ in $\mathrm{Pb}-\mathrm{Pb}$ collisions), the ITS needs to be upgraded to strongly improve the tracking efficiency and the impact parameter resolution at very low transverse momenta.

The main limits of the current setup are given by a SDD maximum readout rate of $1 \mathrm{kHz}$, a material budget of $1.1 \%$ of the radiation length $\left(\mathrm{X}_{0}\right)$ per layer and a spatial resolution of about $120 \mu \mathrm{m}$ at $p_{T}=500 \mathrm{MeV} / c$.

The new ITS will be equipped with seven cylindrical and concentric layers of silicon Monolithic Active Pixel Sensors (MAPS) called ALPIDE (ALice PIxel DEtector). The new ITS apparatus will be divided into an Inner Barrel (IB) containing the three Inner Layers (IL) and an Outer Barrel (OB) containing two Middle and two Outer Layers (ML and OL, respectively).

The layout of the new ITS is sketched in Fig. 1. The total active area will be of about $10 \mathrm{~m}^{2}$. Thanks to a new beam pipe with a smaller radius, the radial distance of the layers from the interaction points will be in the range [22; 400] mm with a pseudo-rapidity acceptance of $|\eta|<1.22$. 


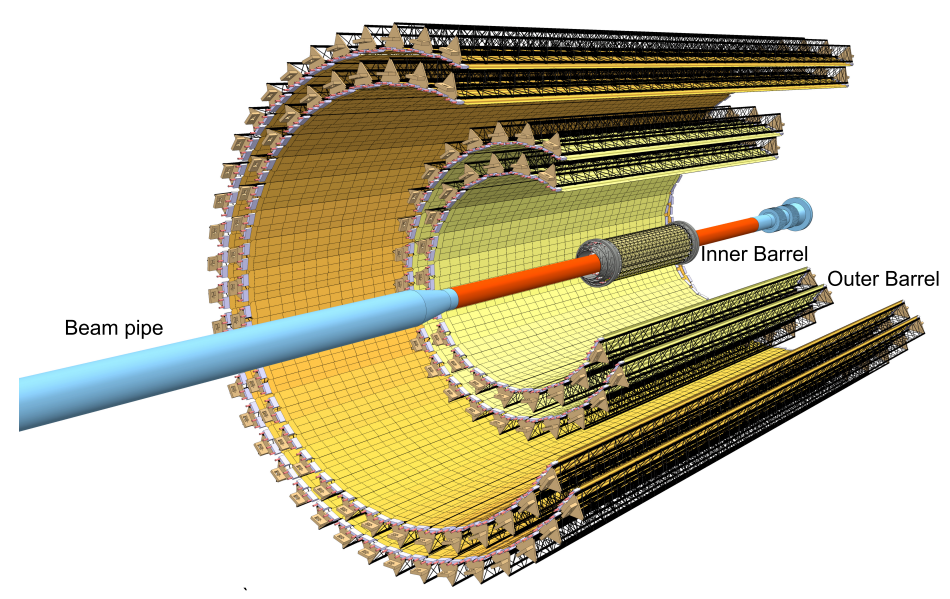

Figure 1: Layout of the new ALICE ITS.

The most important technological features of the upgraded ITS will be:

- a readout rate up to the maximum LHC $\mathrm{Pb}-\mathrm{Pb}$ collision rate of $50 \mathrm{kHz}(400 \mathrm{kHz}$ in pp collisions),

- an improvement of the impact parameter resolution by a factor of $\sim 5$ and $\sim 3$ in $z$ and $r \phi$ direction, respectively, at a transverse momentum $p_{T} \simeq 500 \mathrm{MeV} / c$ (see Fig. 2a). Such an improvement will be possible thanks to

1. a reduced material budget: $0.3 \%(\mathrm{IL})$ and $\sim 1 \%(\mathrm{OL})$ of $\mathrm{X}_{0}$;

2. a reduced pixel size (at present $50 \times 425 \mu \mathrm{m}^{2}$ ) of the order of $30 \times 30 \mu \mathrm{m}^{2}$;

3. the possibily to get closer to the collision vertex.

- An improvement of the tracking efficiency (see Fig. 2b) and $p_{T}$ resolution at low $p_{T}$ thanks to an increased granularity.

\section{ALPIDE chip}

As seen in Sec. 2, the new ITS will be totally equipped with silicon MAPS. They are produced by Towerjazz with its $0.18 \mu \mathrm{m}$ CMOS Imaging process. The chip is composed by a matrix of $512 \times 1024$ pixels with a dimension of $\sim 27 \times 29 \mu \mathrm{m}^{2}$. The overall chip dimension is $1.5 \times 3.0 \mathrm{~cm}^{2}$. The ALPIDE chip is characterized by some crucial features for the performance of the new ITS (further details can be found in [3] and [4]):

- a high-resistivity $(1 \div 6 \mathrm{k} \Omega \mathrm{cm})$ p-type epitaxial layer (active volume for particle detection);

- a small n-well diode about 100 times smaller than the pixel, leading to a small capacitance of few fF;

- a n-well of p-MOS transistors shielded by a deep p-well permitting a full CMOS circuitry within the active area; 


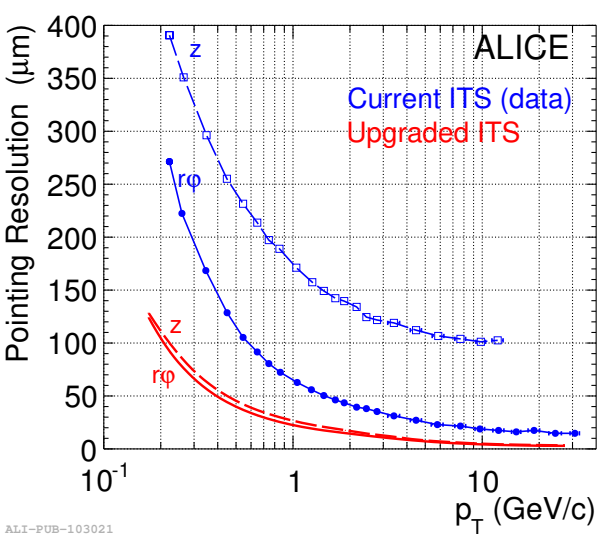

(a) Pointing resolution

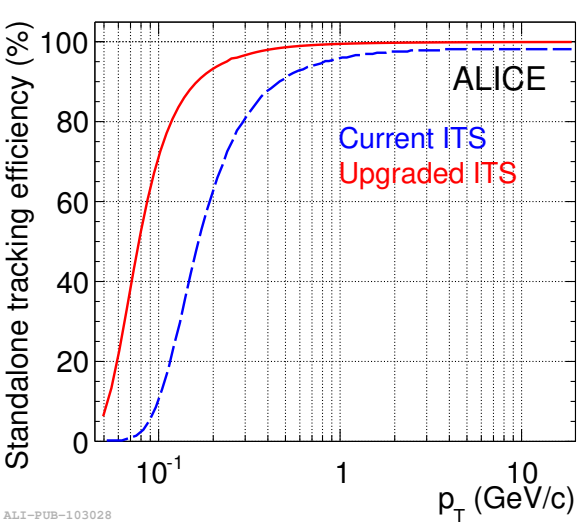

(b) Efficiency

Figure 2: Pointing resolution and efficiency for charged pions as a function of the transverse momentum for the current and upgraded ITS.

- a fast data driven encoder [5] for pixel matrix readout with an integration time of $\sim 2 \mu \mathrm{s}$;

- a pre-amplifier and a discriminator at a pixel level reaching a power consumption lower than $40 \mathrm{~mW} / \mathrm{cm}^{2}$.

The detection efficiency and the fake-hit rate (per event and per pixel) of irradiated and nonirradiated ALPIDE chips were measured in various beam tests and the related results as a function of the threshold are shown in Fig. 3. For this example, the measurement was performed at the CERN Proton Synchrotron (PS) using pions at $6 \mathrm{GeV} / \mathrm{c}^{2}$. The tested chips were powered at a reversed bias voltage $V_{B B}=-3 \mathrm{~V}$. The results demonstrate a sufficient chip operational margin even after $10 \times$ lifetime Non-Ionizing Energy Loss (NIEL) dose.

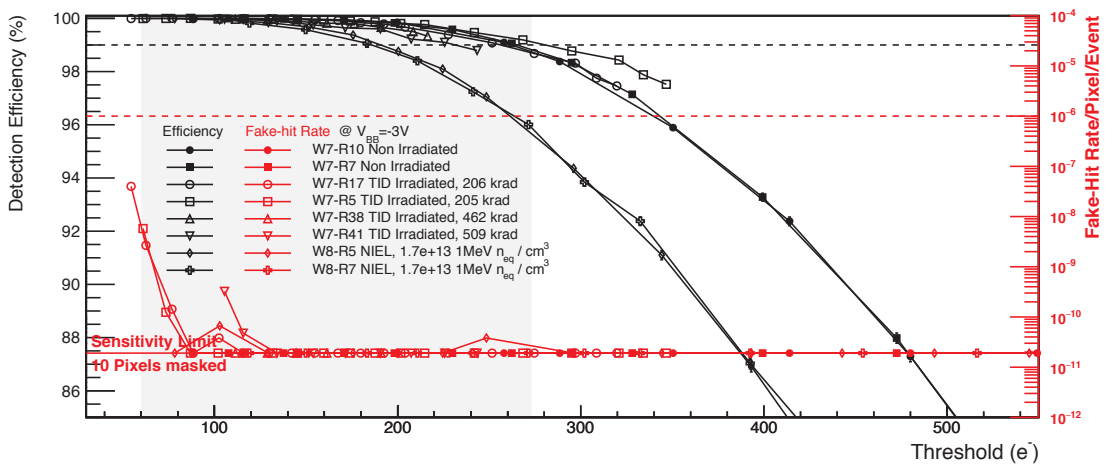

Figure 3: Efficiency and fake-hit rate as a function of the threshold for irradiated and non-irradiated chips.

\section{Stave layout for Inner and Outer Barrels}

The ALPIDE chips described in Sec. 3 are arranged in structures called Modules. A Module of 
the IB and of the OB is made of 9 and 14 chips, respectively. For the OB Modules, a master-slave architecture is implemented. A Flexible Printed Circuit (FPC) wire-bonded to the chips is used for clock, control and data transmission towards and from the outside electronics. On the FPC, an internal bus for master-slave communication is implemented. For more details see [2].

Data are transmitted at a rate of $400 \mathrm{Mb} / \mathrm{s}$ and $1.2 \mathrm{~Gb} / \mathrm{s}$ for the OB and IB Modules, respectively.

The Modules were tested in laboratory measuring an extremely low fake-hit rate lower than $10^{-10}$ /event/pixel compatible with single-chip performance.

The Modules are arranged in longitudinal structures called Staves. An IB Stave is made of one Module, a FPC and a Cold-Plate for chip cooling. An OB Half-Stave (HS) is made of four (seven) Modules for the ML (OL), one FPC per Module, a Power-Bus for chip powering and a Cold Plate. The OB Stave is then formed by two independent Half-Staves. An ultra-light carbon-fiber Space Frame supports the detectors and all the circuitry.

The total lenght of the Staves is about $28 \mathrm{~cm}, 80 \mathrm{~cm}$ and $150 \mathrm{~cm}$ for the IL, ML and OL Staves, respectively. The ITS will be made of 48, 54 and 90 Staves for the IL, ML and OL, respectively.

The Stave structure and components are sketched in Fig. 4.

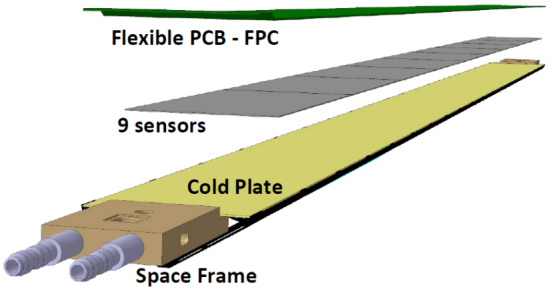

(a) IB Stave

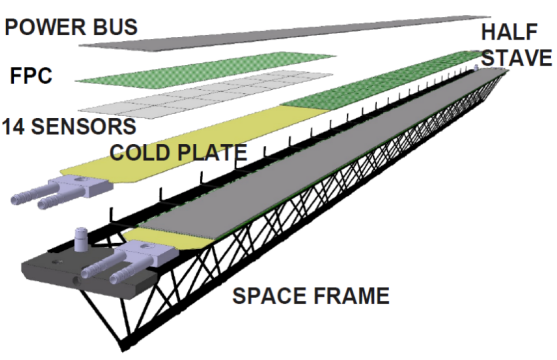

(b) OB Stave

Figure 4: Structure of an IB $(a)$ and OB $(b)$ Stave for the upgraded ALICE ITS.

\section{Expected physics performance ot the upgraded ITS}

In this section, some examples concerning the expected physics performance with the upgraded ITS are presented.

\subsection{Heavy flavour}

The heavy quarks constitute a tagged probe which enables a unique access to their interactions in the QGP [2]. The two main open questions related to heavy-flavour physics are the thermalisation and hadronisation of heavy quarks in the medium and, the heavy quark in-medium energy loss and its mass dependence.

\subsection{1 $B^{+} \rightarrow \bar{D}^{0} \pi^{+}$decay}

With the upgraded ITS the full kinematic reconstruction of beauty hadrons will become accessible. A simulation was performed for $B^{+} \rightarrow \bar{D}^{0}\left(\rightarrow K^{+} \pi^{-}\right) \pi^{+}$. The decay of $B^{+}$has a $c \tau \approx 492 \mu \mathrm{m}$ with a branching ratio (B.R.) of $0.48 \%$. An exclusive reconstruction down to $p_{T}=2 \mathrm{GeV} / c$ will be possible considering the significance and the uncertainties shown in Fig. 5a. 
The combinatorial background reduction will be achieved by selecting pions with $p_{T}>1 \mathrm{GeV} / c$. A further reduction is obtained by recontructing the secondary $\left(B^{+}\right)$and tertiary $\left(\bar{D}^{0}\right)$ vertices. The sensitivity to beauty $v_{2}{ }^{1}$ (elliptic flow) can be seen in Fig. $5 \mathrm{~b}$.

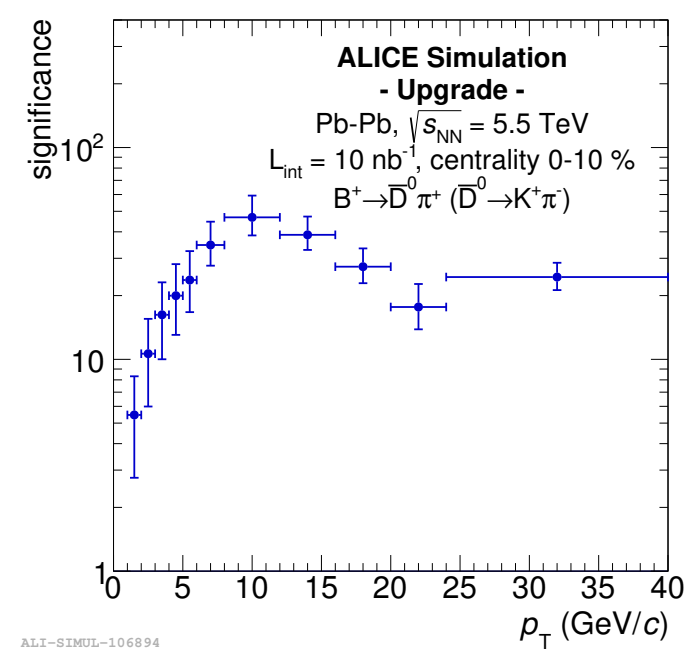

(a) $B^{+}$significance

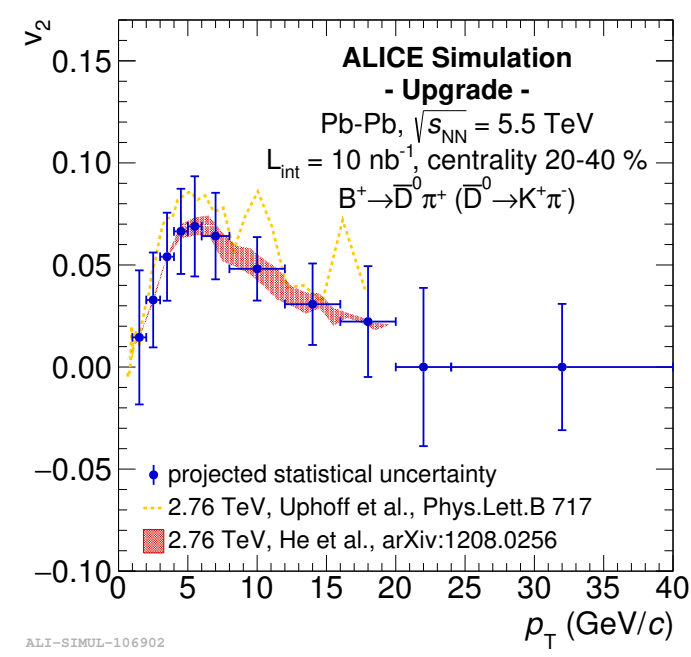

(b) Sensitivity to $v_{2}$

Figure 5: Simulated signficance $(a)$ and $v_{2}$ sensitivity $(b)$ for $B^{+}$mesons in $\mathrm{Pb}-\mathrm{Pb}$ collision at 5.5 $\mathrm{TeV}$.

\subsection{2 $\Lambda_{c}^{+} \rightarrow p K^{-} \pi^{+}$decay}

One of the most challenging heavy-flavour baryon measurement is the $\Lambda_{c}{ }^{+} \rightarrow p K^{-} \pi^{+}$decay with a B.R. of about $5.0 \%$ and a short mean decay lenght of about $60 \mu \mathrm{m}$. Very high tracking precision is needed to separate the decay vertex from the primary vertex [2]. Thanks to the new ITS, a reconstruction of the $\Lambda_{c}{ }^{+}$down to $p_{T}=2 \mathrm{GeV} / c$ will be possible.

Fig. 6a shows the $\mathrm{S} / \mathrm{B}$ ratio for central $\mathrm{Pb}-\mathrm{Pb}$ collisions at $5.5 \mathrm{TeV}$. The $\mathrm{S} / \mathrm{B}$ ratio will improve by a factor 400 in $\mathrm{Pb}-\mathrm{Pb}$ most central collisions at $2<p_{T}<4 \mathrm{GeV} / c$.

In Fig. 6b, the expected $\Lambda_{c}{ }^{+}$nuclear modification factor ${ }^{2}\left(R_{A A}\right)$ for an integrated luminosity of $\mathrm{L}_{\text {int }}$ $=10 \mathrm{nb}^{-1}$ is plotted as a function of $p_{T}$.

\subsection{Low-mass dielectrons}

The electromagnetic radiation is produced at all stages of the collision, and since leptons couple only weakly to the surrounding medium, their spectrum retains information of the entire system evolution [2]. Hence, the main motivations for studying low-mass dielectrons are the measurement of the temperature of the emitting medium and the study of the system space-time evolution.

The enhanced low- $p_{T}$ traking capability will allow us to track electrons down to $p_{T}>50 \mathrm{MeV} / c$. The better impact parameter resolution will enable efficient tagging of electrons from semi-leptonic

\footnotetext{
${ }^{1}$ the second coefficient of the Fourier series expansion of the azimuthal distribution of the number of particles with respect to the reaction plane

${ }^{2} R_{A A}=\frac{1}{\left\langle N_{\text {collisions }}\right\rangle} \frac{d N^{P b-P b} / d p_{T}}{d N^{p p} / d p_{T}}$
} 


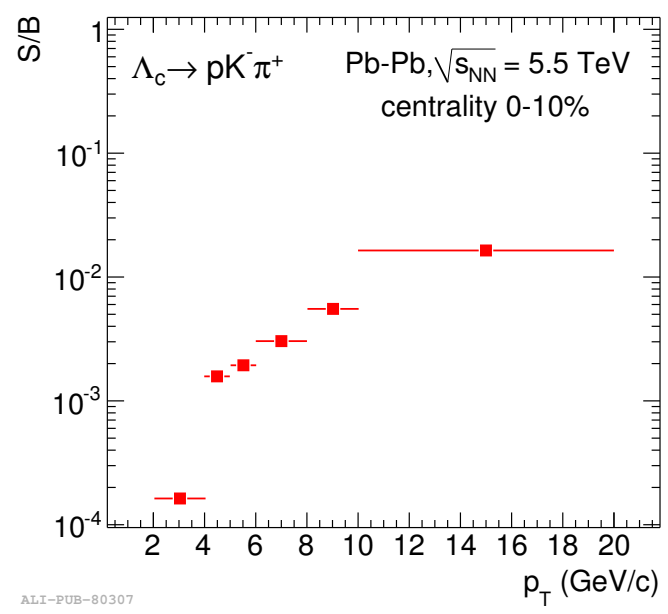

(a) $\Lambda_{c}{ }^{+}$significance

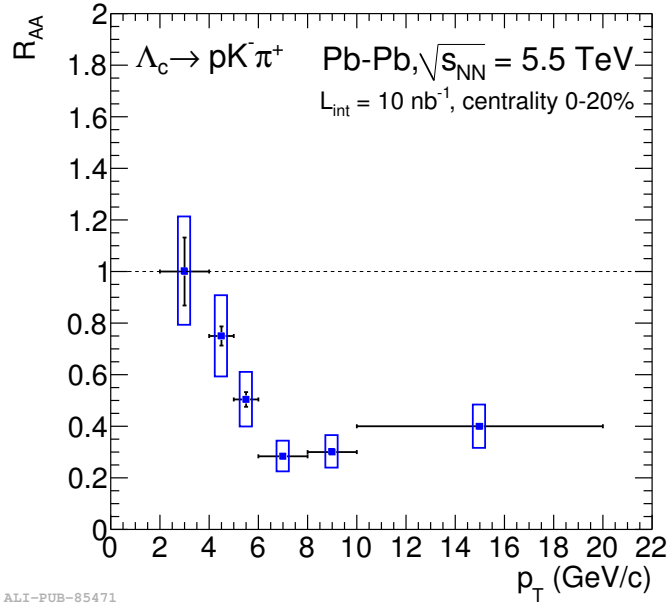

(b) $\Lambda_{c}{ }^{+} R_{A A}$

Figure 6: Expected significance ( $a$ ) and $R_{A A}(b)$ for $\Lambda_{c}{ }^{+}$baryons in $\mathrm{Pb}-\mathrm{Pb}$ central collision at 5.5 $\mathrm{TeV}$.

charm decays, which can be separated from prompt dileptons.

Figure 7a shows the expected dielectron excess yield in $\mathrm{Pb}-\mathrm{Pb}$ collisions at $\sqrt{s_{N N}}=5.5 \mathrm{TeV}$ after hadronic cocktail subtraction using the current ALICE setup [6] [1]. The same is shown in Fig. 7b, but with the upgraded ITS and TPC [6].

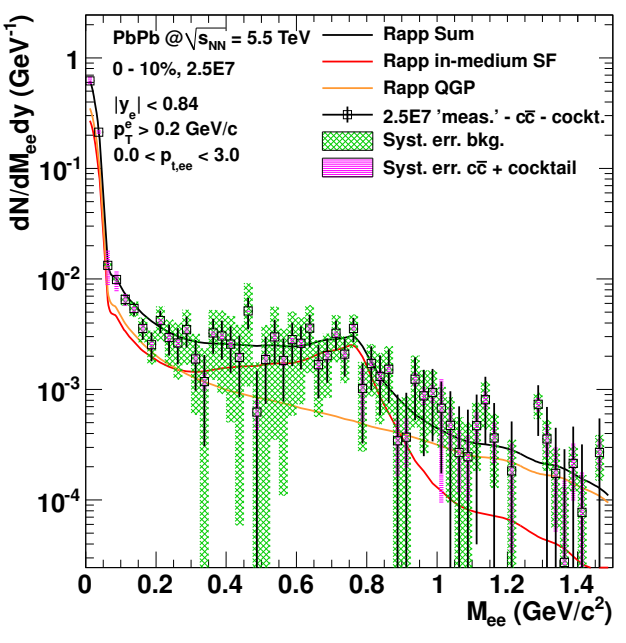

(a) With current ALICE setup

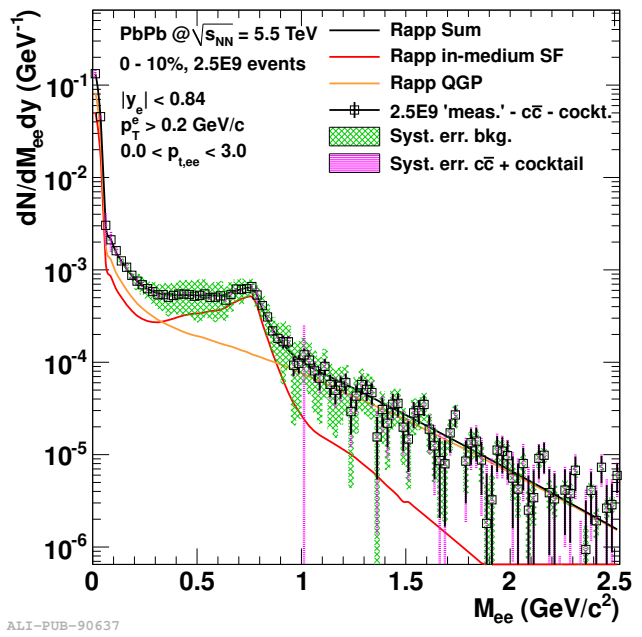

(b) With upgraded ITS and TPC

Figure 7: Expected dielectron excess yield in $\mathrm{Pb}-\mathrm{Pb}$ collisions at $\sqrt{s_{N N}}=5.5 \mathrm{TeV}$ after hadronic cocktail subtraction using the current ALICE setup $(a)$ and the upgraded ITS and TPC $(b)$. The reduced statistical and systematic uncertainties make a temperature measurement in the intermediatemass region feasible. For further details see [6] and [1]. 


\section{Summary and outlook}

The present ITS will be upgraded in 2019-2020 during the Long Shutdown 2 of the LHC in order to improve the tracking efficiency and impact parameter resolution at very low transverse momenta, as well as the read-out rate capabilities. The new layout will consists of seven cylindrical and concentric layers of more than 24000 silicon Monolithic Active Pixel Sensors (MAPS) called ALPIDE, produced with the $0.18 \mu \mathrm{m}$ CMOS Imaging process of Towerjazz. They are characterized by a matrix of $512 \times 1024$ pixels readout with a fast data driven encoder.

The physics performance will be strongly improved giving access to precise measurement of heavyflavour baryons and mesons and, to low-mass dileptons. More precise measurements of thermalisation and recombination of heavy-flavour inside the Quark Gluon Plasma together with its temperature and its space-time evolution will be perfomed.

The production of all detector elements, including auxiliary systems, will enter full swing in 2017 and will be completed by the end 2018. After commissioning in the assembly hall, the detector will be installed in the experiment during the second half of 2020.

\section{Aknowledgements}

The ITS project acknowledges the support received from several test beam facilities during the R\&D phase: BTF (Frascati, Italy), PS \& SPS (CERN), DESY TB (Hamburg, Germany), PAL (Pohang, Korea), SLRI (Nakhon Ratchasima, Thailand). In particular the availability of beam at BTF and DESY TB, along with the precious help from local staff, during the Long Shutdown 1 of the CERN accelerator complex, has been instrumental to the development of the ALPIDE sensor and to keep the project on schedule.

The final ALPIDE chip as well as several prototype versions have been tested for TID and NIEL hardness at the following irradiation facilities: BASE LBL (Berkeley,US), HIF UC (Louvain-laNeuve, Belgium), NPI (Prague, Cech Republic), TRIGA Mark II reactor (Ljubljana, Slovenia).

\section{References}

[1] ALICE Collaboration, Upgrade of the ALICE Experiment: Letter of Intent, J. Phys. G 41 (2014) 087001.

[2] ALICE Collaboration, Technical Design Report for the Upgrade of the ALICE Inner Tracking System, J. Phys. G 41 (2014) 087002.

[3] Aglieri Rinella G. et al.,The ALPIDE pixel sensor chip for the upgrade of the ALICE Inner Tracking System, NIM A 845 (2017) 583-587.

[4] Šljić M. et al., ALPIDE: the Monolithic Active Pixel Sensor for the ALICE ITS upgrade, 2016 JINST 11 C 11025

[5] P. Yang et al., Low-power priority Address-Encoder and Reset-Decoder data-driven readout for Monolithic Active Pixel Sensors for tracker system, Nucl. Instrum. Meth. A 785 (2015) 61.

[6] P. Reichelt (ALICE Collaboration), Low-Mass Dielectron Production in pp, $\mathrm{p}-\mathrm{Pb}$ and $\mathrm{Pb}-\mathrm{Pb}$ Collisions with ALICE, J.Phys.Conf.Ser. 612 (2015) no.1, 012028. 